\title{
Coincidence and some systems of inequalities.
}

\author{
By Hukukane NiKAIDÔ
}

(Received April 27, 1959)

\section{Introduction}

The use of a fixed point theorem in the study of systems of inequalities originated with von Neumann's works $[\mathbf{2 1}, \mathbf{2 2}]$ on his minimax theorem. Since then, problems of similar line have attracted broad attention, and remarkable progress has been made in the generalization of the results as well as the improvement of the methods of proof.

The method of von Neumann in [22] was simplified and reformulated a few years later as Kakutani's fixed point theorem [12] which is very useful and frequently used in dealing with many similar problems. The development of topological devices in the direction initiated by Kakutani now culminates in the results of Eilenberg-Montgomery [6] and Begle [2] which extend the noted Lefschetz's trace formula $[\mathbf{1 6}, \mathbf{1 7}]$ to set-valued mappings. On the other hand improvement has also been made in the effort to treat the problems with a minimum amount of topology. Numerous proofs ${ }^{1)}$ based on the separation of a convex set by a hyperplane, i. e. those appealing to several types of Hahn-Banach's theorem have appeared to meet the most elementary cases of the minimax theorem or its variants, since the first such simplification was made by von Neumann himself [23]. Beside this line of simplification, this writer, following and pushing over the idea in Nash's result [20], showed that Brouwer's fixed point theorem can, with the aid of a kind of Kuratowski map technique, directly apply to general minimax or equilibrium point theorems that cannot be handled in the standard way as established by Kakutani because of the lack of the continuity of relevant functions. Our way of reducing the resolution of a problem to a fixed point problem is, we believe, of interest, in that it utilizes the potentiality possessed by the fixed point theorem concerned more fully than that in the standard line does.

Meanwhile, since the von Neumann's initial work attention had mainly been confined to some game problems or their variants, and no attack had ever been made against relevant conjectures in the orthodox mathematical economics until in recent times Arrow-Debreu [1], McKenzie [18], Gale [10]

1) Without claim for completeness we cite a few examples $[\mathbf{7}, \mathbf{8}, \mathbf{1 1}, \mathbf{1 4}, \mathbf{1 5}, \mathbf{2 7}]$. 
and this writer [26] independently and almost simultaneously gave reformulations and proofs to the most basic conjecture in the theory of general economic equilibrium as founded by L. Walras around the end of the last century. Here as in the theory of games fixed point theorems or their equivalent propositions proved to be very powerful. It is interesting as well as significant that the minimax problems and those of economic equilibrium have some intersection in common and reveal a certain similarity between them.

The present paper offers a theorem on the existence of solutions for some system of inequalities with the intention to generalize and to give a unified treatment to the results of Gale and this writer regarding the existence of economic equilibrium on one hand, and the theorems in game problems including this writer's previous results on the other. The theorem will be stated and proved in section 4. It needs a certain knowledge of Vietoris homology theory for compact Hausdorff spaces in its statement and proof. Section 2 is preliminary in nature and provides with some of basic results in the Vietoris homology theory which will be used later on. The proof of the main theorem will be done by further improving the method in the previous papers $[\mathbf{2 4}, \mathbf{2 5}]$ and relating the problem to a special case of EilenbergMontgomery-Begle's coincidence theorem $[\mathbf{2 , 6}$. In view of the much elementary nature of this special coincidence theorem, a simple alternative proof will be given to it by establishing an analogue of Sperner's lemma.

Section 5 will be devoted to a reformulation of a part of the main results in locally convex topological linear spaces in the hope to bring a duality relation behind the theorem into relief.

Finally it is acknowledged that we are greatly indebted to Prof. S. Seki for his invaluable comment and suggestions.

\section{Vietoris cycles.}

In this section basic concepts and elementary results in the Vietoris homology theory will be recalled and enumerated for subsequent use. Unless otherwise stated, coefficients are always elements of a fixed field $F$.

2.1. $V$-cycles. In the sequel a covering always means a finite covering by open sets. Let $M, N, \cdots$ be coverings of a compact Hausdorff space $S$. For two coverings $M$ and $N$ of $S, M>N$ means, by definition, that $M$ refines $N$. Thus the totality of all coverings of $S$ is thought of as being directed by means of this partial order.

An oriented $n$-simplex $\sigma$ in $S$, by definition, is simply an ordered collection of $n+1$ points $x_{0}, x_{1}, \cdots, x_{n}$, of $S$ up to their even permutations; these 
points are called the vertices of $\sigma$. We write $\sigma=x_{0} x_{1} \cdots x_{n}$ whereas by $[\sigma]$ is meant the point set of all the vertices of $\sigma$. For a covering $M$, we denote by $K(M)$ the simplicial complex formed by all oriented simplexes $\sigma$ of $S$ such that $[\sigma]$ is included in some member of $M$. Whenever preferable, these simplexes will be called $M$-simplexes. Therefore, if $M>N, K(M)$ is a subcomplex of $K(N)$. We always use finite chains in $K(M)$ over a field $F$. For a chain $c=\Sigma \alpha_{j} \sigma_{j}$ in $K(M),[c]$ stands for the union of $\left[\sigma_{j}\right]$ for all $\sigma_{j}$ with $\alpha_{j} \neq 0$ in $c$.

An $n$-dimensional Vietoris cycle $z^{n}=\left\{z^{n}(M)\right\}$, an $n$-V-cycle in short, is a collection of $n$-cycles of $S$, each $z^{n}(M)$ being an $n$-cycle in $K(M)$, such that $M>N$ implies $z^{n}(M) \sim z^{n}(N)$ in $K(N)$. Two $n$-V-cycles $z_{1}{ }^{n}=\left\{z_{1}{ }^{n}(M)\right\}$ and $z_{2}^{n}=\left\{z_{2}^{n}(M)\right\}$ add up to an $n$ - $V$-cycle $z^{n}=\left\{z^{n}(M)\right\}$ by the formula $z^{n}(M)=$ $z_{1}^{n}(M)+z_{2}^{n}(M)$. With this addition the totality of all $n$ - $V$-cycles becomes a vector space $Z^{n}(S)$ over $F$, the group of $n$ - $V$-cycles. An $n$-V-cycle $z^{n}=\left\{z^{n}(M)\right\}$ is said to bound, or to be homologous to zero, $z^{n} \sim 0$ in symbol, whenever each $z^{n}(M) \sim 0$ in $K(M)$. All these $n$ - $V$-cycles form a vector subspace $B^{n}(S)$ of $Z^{n}(S)$, the group of all bounding $n$-V-cycles. The factor group $H^{n}(S)=$ $Z^{n}(S) / B^{n}(S)$ is, by definition, the $n$-dimensional Vietoris homology group. Incidentally, we adopt augumented homology groups so that $0-V$-cycles are restricted to those whose coordinates cycles $z^{0}(M)$ have the Kronecker index 0.

It is known (see $[3, \mathrm{pp} .536-7]$ ) that $H^{n}(S)$ is isomorphic to the $n$-dimensional Čech homology group defined by means of the nerves of coverings.

\subsection{Some theorems.}

A compact Hausdorff space is called acyclic, if all its homology groups vanish. Contractible spaces are well-known acyclic spaces; convex sets are much special cases of the contractible spaces. The following important theorem due to Vietoris is most basic throughout this paper as in those of Eilenberg-Montgomery [6] and Begle [2].

ThEOREM 1 (Vietoris [31], Begle [3]).

Let $S$ and $T$ be two compact Hausdorff spaces, and $f: S \rightarrow T$ a continuous map onto $T$ such that the inverse image $f^{-1}(q)$ of each $q \in T$ is acyclic. (We call such a map a Vietoris map.) Under these circumstances $f$ induces isomorphisms onto, between $H^{n}(S)$ and $H^{n}(T)$, for any dimensions.

For its proof the well-exploring Begle's paper [3] may be consulted.

Also the following results are important to the discussion in subsequent sections.

Theorem 2. Let $S$ be a compact Hausdorff space which is acyclic. Then for any covering $N$ there is a refinement $M$ such that every n-cycle $r$ in $K(M)$ bounds a chain in $K(N)$. 
A proof is given to this result by Begle [3] based on the relationships between $V$-cycles and Čech cycles and on a proposition regarding essential elements in an inverse system of finite-dimensional vector spaces (Lefschetz $[17$, p. $79,(27.12)$ and (27. 13)]). It is also noted that the restriction of the group of coefficients to a field is made only in connection to Theorem 2.

This theorem can be put in a more useful form. To this end we consider relations of coverings of a space to those of its subspaces. Let $S$ be a Hausdorff space and $T$ a closed subspace of $S$. For a covering $N$ of $S$ let $K(T, N)$ stand for the subcomplex of $K(N)$, formed by all $\sigma$ with $[\sigma] \subset T$. In contrast with this notation, $K_{T}\left(N^{\prime}\right)$ will be used to designate the complex formed by all $N^{\prime}$-simplexes in $T$, where $N^{\prime}$ is a covering of $T$. A covering $N$ of $S$ induces a covering $N^{\prime}$ of $T$ in the natural fashion. Coversely, a covering $N^{\prime}$ of $T$ may be thought of as induced by some covering $N$ of $S$, since $T$ is closed in $S$. Now assume that $T$ is compact and acyclic. Given any covering $N$ of $S$, then $K_{T}\left(N^{\prime}\right)=K(T, N)$, where $N^{\prime}$ is the covering induced by $N$ in $T$. By Theorem 2 there is a refinement $M^{\prime}$ of $N^{\prime}$ such that every $n$-cycle in $K_{T}\left(M^{\prime}\right)$ bounds a chain in $K_{T}\left(N^{\prime}\right)$. Let $M$ be one covering of $S$ that induces $M^{\prime}$ in $T$, and take a common refinement $L$ of $M$ and $N$. Then, as $L$ induces a covering in $T$ finer than $M^{\prime}$, every $n$-cycle in $K(T, L)$ bounds a chain in $K(T, N)$.

We state this result in a more general way as follows:

Corollary. Let $S$ be a Hausdorff space and $T_{i}$ compact acyclic subsets of $S$, finite in number. Given any covering $N$ of $S$, then there is a refinement $L$ of $N$ such that for each $i$ every n-cycle in $K\left(T_{i}, L\right)$ bounds a chain in $K\left(T_{i}, N\right)$.

Proof. In view of the above discussion, for each $i$ choose one covering $L_{i}$ which has the desired property just for $T_{i}$. A covering $L$ sought for will be obtained by taking a common refinement of all $L_{i}$.

\section{An analogue of Sperner's lemma.}

3.1. Let $S$ be a compact, Hausdorff space and $Y$ an $n$-dimensional Euclidean simplex whose vertices are $a_{i}(i=0,1, \cdots, n)$. Let furthermore $f$ be a continuous onto-map: $S \rightarrow Y$. Whenever a $k$-dimensional face $a_{i_{0}} a_{i_{1}} \cdots a_{i_{k}}$ of $Y$ is viewed as a point set, it will be denoted by $\left|a_{i_{0}} a_{i_{1}} \cdots a_{i_{k}}\right|$. The inverse image $f^{-1}\left(\left|a_{i_{0}} a_{i_{1}} \cdots a_{i_{k}}\right|\right)$ of a $k$-dimensional face $\left|a_{i_{0}} a_{i_{1}} \cdots a_{i_{k}}\right|$ of $Y$ will be called a $k$-face of $S$. For any point $p \in S$ there obviously exists one and only one face of the lowest $k$ which we call the carrier of $p$.

Let $L$ stand for the simplicial complex formed by all faces of $Y . K(N)$ has the same meaning as in the foregoing section. All chains appearing are finite chains over a field $F$. Suppose that there be given a chain homomor- 
phism $\tau$ which fulfills the following conditions:

(1) $\tau\left(c^{k}\right)$ is a $k$-chain in $K(N)$ for each $k$-chain $c^{k}$ in $L$.

(2) $\tau$ commutes with the boundary operator $\partial$.

(3) $\left[\tau\left(a_{i_{0}} \alpha_{i_{1}} \cdots a_{i_{k}}\right)\right] \subset f^{-1}\left(\left|a_{i_{0}} a_{i_{1}} \cdots a_{i_{k}}\right|\right)$ for any face $a_{i_{0}} a_{i_{1}} \cdots a_{i_{k}}$ of $Y$.

(4) $\tau\left(a_{i}\right)$ is a single vertex for each vertex $a_{i}$.

It will later be shown that $\tau$ can actually be given under certain conditions.

Furthermore suppose that there be given a vertex assignment $p \rightarrow \omega(p)$ which assigns to each vertex $p$ of $K(M)$ a vertex of $L$ in such a way that if $f^{-1}\left(\left|a_{i_{0}} a_{i_{1}} \cdots a_{i_{k}}\right|\right)$ is the carrier of $p$, then $\omega(p)$ is a vertex of $a_{i_{0}} a_{i_{1}} \cdots a_{i_{k}}$.

We next define a $\operatorname{sign} \varepsilon\left(\sigma^{n}\right)$ of an $n$-simplex $\sigma^{n}$ in $K(M) . \omega$ is clearly a simplicial map and induces a chain homomorphism which we also denote by $\omega . \varepsilon\left(\sigma^{n}\right)$ is defined as follows:

$$
\varepsilon\left(\sigma^{n}\right)=\left\{\begin{array}{rll}
1 & \text { if } & \omega\left(\sigma^{n}\right)=a_{0} \alpha_{1} \cdots a_{n} \\
-1 & \text { if } \quad \omega\left(\sigma^{n}\right)=-a_{0} a_{1} \cdots a_{n} \\
0 & \text { otherwise. }
\end{array}\right.
$$

If $\varepsilon\left(\sigma^{n}\right) \neq 0, \sigma^{n}$ will be called regular. With this setup in mind, we shall prove

Lemma 1. Let $\tau\left(a_{0} a_{1} \cdots a_{n}\right)=\sum \alpha_{j} \sigma_{j}^{n}$, where $\tau$ is a chain homomorphism as expounded above. Then we have $\sum \alpha_{j} \varepsilon\left(\sigma_{j}{ }^{n}\right) \neq 0$. This implies, in particular, the existence of at least one regular n-simplex.

PROOF. The proof will be done by induction over $n$ as in the classical lemma of Sperner. We carry the definition of $\varepsilon$ to $(n-1)$-simplexes in $K(N)$ in the following natural way.

$$
\varepsilon\left(\sigma^{n-1}\right)=\left\{\begin{array}{rll}
1 & \text { if } \quad \omega\left(\sigma^{n-1}\right)=a_{1} a_{2} \cdots a_{n} \\
-1 & \text { if } \quad \omega\left(\sigma^{n-1}\right)=-a_{1} a_{2} \cdots a_{n} \\
0 & \text { otherwise. }
\end{array}\right.
$$

An $(n-1)$-dimensional face $\sigma^{n-1}$ of an $n$-simplex $\sigma^{n}$ in $K(N)$ will be called a regular face, if $\varepsilon\left(\sigma^{n-1}\right) \neq 0$. Put $\xi\left(\sigma^{n}\right)=\sum_{k}\left[\sigma_{k}^{n-1}: \sigma^{n}\right] \varepsilon\left(\sigma_{k}^{n-1}\right)$, where $[:]$ stands for the incidence number. Then it is immediate that

$$
\sum_{j} \alpha_{j} \xi\left(\sigma_{j}^{n}\right)=\sum_{k} \varepsilon\left(\sigma_{k}{ }^{n-1}\right) \sum_{j} \alpha_{j}\left[\sigma_{k}{ }^{n-1}: \sigma_{j}{ }^{n}\right] \text {. }
$$

Also in view of the assumptions on $\tau$ we immediately get ${ }^{2)}$

$$
\begin{aligned}
& \partial \tau\left(a_{0} a_{1} \cdots a_{n}\right)=\sum_{i}(-1)^{i} \tau\left(a_{0} \cdots \hat{a}_{i} \cdots a_{n}\right), \\
& \partial \tau\left(a_{0} a_{1} \cdots a_{n}\right)=\sum_{k} \sum_{j} \alpha_{j}\left[\sigma_{k}{ }^{n-1}: \sigma_{j}{ }^{n}\right] \sigma_{k}{ }^{n-1} .
\end{aligned}
$$

2) In the sequel a circumflex over a letter will be used to mean that this letter does not appear in the formula concerned. 
Now let $\tau\left(a_{1} a_{2} \cdots a_{n}\right)=\sum_{k} \beta_{k} \sigma_{k}{ }^{n-1}$. It will be shown by making use of these relations that

$$
\sum_{j} \alpha_{j} \varepsilon\left(\sigma_{j}{ }^{n}\right)=\sum_{k} \beta_{k} \varepsilon\left(\sigma_{k}{ }^{n-1}\right) .
$$

First, if $\varepsilon\left(\sigma_{k}^{n-1}\right)=0$, clearly $\beta_{k} \varepsilon\left({\sigma_{k}}^{n-1}\right)=\varepsilon\left(\sigma_{k}{ }^{n-1}\right) \sum_{j} \alpha_{j}\left[\sigma_{k}{ }^{n-1}: \sigma_{j}{ }^{n}\right]$. Next suppose $\varepsilon\left(\sigma_{k}{ }^{n-1}\right) \neq 0$. In this case, from the definition of $\varepsilon$ it follows by (3) that $\sigma_{k}{ }^{n-1}$ appears in no chains $\tau\left(a_{0} \cdots \hat{\alpha}_{i} \cdots a_{n}\right)(i \neq 0)$. Hence its coefficient $\sum_{j} \alpha_{j}\left[\sigma_{k}{ }^{n-1}: \sigma_{j}{ }^{n}\right]$ in $\partial \tau\left(a_{0} \alpha_{1} \cdots a_{n}\right)$ must equal $\beta_{k}$ with which it appears in $\tau\left(a_{1} a_{2} \cdots a_{n}\right)$. We accordingly have $\beta_{k} \varepsilon\left(\sigma_{k}{ }^{n-1}\right)=\varepsilon\left(\sigma_{k}{ }^{n-1}\right) \sum_{j} \alpha_{j}\left[\sigma_{k}{ }^{n-1}: \sigma_{j}{ }^{n}\right]$ in any case. It is noted that the above argument was done regardless of whether $\beta_{k}=0$ or not. We have thereby proved $\sum_{j} \alpha_{j} \xi\left(\sigma_{j}{ }^{n}\right)=\sum_{k} \beta_{k} \varepsilon\left(\sigma_{k}{ }^{n-1}\right)$. It remains to show that $\xi\left(\sigma_{j}{ }^{n}\right)$ $=\varepsilon\left(\sigma_{j}{ }^{n}\right)$. First we consider the case where $\sigma_{j}{ }^{n}$ is regular. In this case we have $\omega\left(\sigma_{j}^{n}\right)=\varepsilon\left(\sigma_{j}^{n}\right) a_{0} a_{1} \cdots a_{n}$. Let $\sigma_{j}^{n}=p_{0} p_{1} \cdots p_{n}$. Then $\sigma_{j}^{n}=\varepsilon\left(\sigma_{j}{ }^{n}\right) p_{i_{0}} p_{i_{1}} \cdots p_{i_{n}}$ where $\omega\left(p_{i_{\nu}}\right)=\alpha_{\nu} . \quad \sigma_{j}^{n}$ clearly has just one regular face $\sigma_{k}{ }^{n-1}$ so that $\sigma_{k}{ }^{n-1}=$ $\varepsilon\left(\sigma_{k}{ }^{n-1}\right) p_{i_{1}} p_{i_{3}} \cdots p_{i_{n}}$. Hence $\xi\left(\sigma_{j}{ }^{n}\right)=\left[\varepsilon\left(\sigma_{k}{ }^{n-1}\right) p_{i_{1}} p_{i_{2}} \cdots p_{i_{n}}: \varepsilon\left(\sigma_{j}{ }^{n}\right) p_{i_{0}} p_{i_{1}} \cdots p_{i_{n}}\right] \varepsilon\left(\sigma_{k}{ }^{n-1}\right)=$ $\varepsilon\left(\sigma_{j}^{n}\right)$. Next suppose that $\sigma_{j}^{n}$ is not regular. For an obvious reason we have only to discuss the case where $\sigma_{j}{ }^{n}$ has regular faces. Let $\sigma_{j}{ }^{n}=$ $\gamma \cdot p_{0} p_{1} \cdots p_{n}, \gamma= \pm 1, \omega\left(p_{i}\right)=\alpha_{i}(1 \leqq i \leqq n), \omega\left(p_{0}\right)=a_{\nu}$ for some $\nu$ with $1 \leqq \nu \leqq n$. There are just two regular faces, $\sigma_{l}{ }^{n-1}$ and $\sigma_{k}{ }^{n-1}$. They may be written as $\sigma_{l}^{n-1}=\varepsilon\left(\sigma_{l}^{n-1}\right) p_{1} p_{2} \cdots p_{n}, \sigma_{k}{ }^{n-1}=(-1)^{\nu-1} \varepsilon\left(\sigma_{k}{ }^{n-1}\right) p_{0} p_{1} \cdots \hat{p}_{\nu} \cdots p_{n}$. Accordingly, $\xi\left(\sigma_{j}{ }^{n}\right)=$ $\left[\varepsilon\left(\sigma_{l}^{n-1}\right) p_{1} p_{2} \cdots p_{n}: \gamma p_{0} p_{1} \cdots p_{n}\right] \varepsilon\left(\sigma_{l}{ }^{n-1}\right)+\left[(-1)^{\nu-1} \varepsilon\left(\sigma_{k}{ }^{n-1}\right) p_{0} p_{1} \cdots \hat{p}_{\nu} \cdots p_{n}: \gamma p_{0} p_{1} \cdots p_{n}\right]$ $\varepsilon\left(\sigma_{k}{ }^{n-1}\right)=\gamma(1-1)=0=\varepsilon\left(\sigma_{j}{ }^{n}\right)$. Therefore always $\xi\left(\sigma_{j}{ }^{n}\right)=\varepsilon\left(\sigma_{j}{ }^{n}\right)$. We have thereby showed that

$$
\sum_{j} \alpha_{j} \varepsilon\left(\sigma_{j}{ }^{n}\right)=\sum_{k} \beta_{k} \varepsilon\left(\sigma_{k}^{n-1}\right) .
$$

The lemma is clearly true for $n=0$. Suppose that it is true for $n-1$. Then, on confining $f$ to $f^{-1}\left(\left|a_{1} a_{2} \cdots a_{n}\right|\right)$ and $\tau$ to $a_{1} a_{2} \cdots a_{n}$, we can therefore see that $\sum_{j} \alpha_{j} \varepsilon\left(\sigma_{j}{ }^{n}\right)=\sum_{k} \beta_{k} \varepsilon\left(\sigma_{k}{ }^{n-1}\right) \neq 0$, which proves the lemma for $n$.

\subsection{Construction of $\tau$.}

Here the actual construction of $\tau$ will be made under certain assumptions by following the procedure of Eilenberg and Montgomery [6, p. 218] for compacta, and adapting it to the present formulation.

We henceforth assume that $f: S \rightarrow Y$ is a Vietoris map. As $Y$ is acyclic, so is $S$ by Theorem 1. Also, from the acyclicity of faces of $Y$ it follows that any $k$-face $T\left(i_{0}, i_{1}, \cdots, i_{k}\right)=f^{-1}\left(\left|a_{i_{0}} a_{i_{1}} \cdots a_{i_{k}}\right|\right)$ are acyclic $(k=0,1, \cdots, n)$.

Lemma 2. If $f$ is a Vietoris map, for any covering $N$ there is $a$, with prescribed $\tau\left(a_{i}\right)$, that satisfies (1)-(4) in 3.1.

PROOF. By a repeated application of the corollary to Theorem 2, we can take a sequence of coverings $M_{0}>M_{1}>\cdots>M_{n-1}>M_{n}=N$ such that for each $(k+1)$-face $T\left(i_{0}, i_{1}, \cdots, i_{k+1}\right)$ every $k$-cycle in $K\left(T\left(i_{0}, i_{1}, \cdots, i_{k+1}\right), M_{k}\right)$ bounds a chain 
in $K\left(T\left(i_{0}, i_{1}, \cdots, i_{k+1}\right), M_{k+1}\right),(k=0,1, \cdots, n-1)$. First, we construct $\tau$ for all 0 chains in $L$, by linearly extending the prescribed values $\tau\left(a_{i}\right) \in f^{-1}\left(\alpha_{i}\right)$ onto all 0-chains. Suppose that the construction has been carried out up to $k$ dimension in such a way that $\tau\left(\sigma^{m}\right)$ is an $m$-chain in $K\left(f^{-1}\left(\left|\sigma^{m}\right|\right), M_{m}\right)$ for any $m$-simplex $\sigma^{m}$ of $L, m \leqq k$. Take any $(k+1)$-simplex $\sigma^{k+1}=a_{i_{0}} a_{i_{1}} \cdots a_{i_{k+1}}$. Then $\tau\left(\partial \sigma^{k+1}\right)$ is a well-defined $k$-chain in $K\left(f^{-1}\left(\left|\sigma^{k+1}\right|\right), M_{k}\right)$. But $\partial \tau\left(\partial \sigma^{k+1}\right)=\tau\left(\partial \partial \sigma^{k+1}\right)$ $=0$ so that $\tau\left(\partial \sigma^{k+1}\right)$ is a $k$-cycle in $K\left(f^{-1}\left(\left|\sigma^{k+1}\right|\right), M_{k}\right)$. In view of the relationship between $M_{k}$ and $M_{k+1}, \tau\left(\partial \sigma^{k+1}\right)$ bounds a $(k+1)$-chain in $K\left(f^{-1}\left(\left|\sigma^{k+1}\right|\right), M_{k+1}\right)$, which will be taken to be $\tau\left(\sigma^{k+1}\right)$. The construction for dimension $k+1$ will be completed by linearly extending $\tau\left(\sigma^{k+1}\right)$ onto all $(k+1)$-chains in $L$.

\subsection{Existence of coincidence.}

An immediate consequence of the results obtained above is the following coincidence theorem, which, though a special case of Eilenberg-MontgomeryBegle's theorem $[\mathbf{2 , 6}$, will be given here a simple direct proof, in that we need only this special type of coincidence in the subsequent discussion.

Theorem 3. Let $S$ be a compact Hausdorff space and $Y$ a finite-dimensional compact convex set. Suppose that there be two given continuous maps $f, \theta: S \rightarrow Y$ one of which, say $f$, is a Vietoris map. Then at some point $\bar{p} \in S$ coincidence occurs, i.e., $f(\bar{p})=\theta(\bar{p})$.

Proof. Since $Y$ is a finite-dimensional compact convex set, it may be assumed to be a Euclidean $n$-simplex. Let $f_{i}(p), \theta_{i}(p)$ respectively stand for the $i$-th barycentric coordinates of $f(p), \theta(p)$. Define $n+1$ closed subsets $F_{i}$ of $S$ by $F_{i}=\left\{p \mid p \in S, f_{i}(p) \geqq \theta_{i}(p)\right\}$. As in the classical Knaster-KuratowskiMazurkiewicz's proof of Brouwer's theorem [13], it is immediate to see that $f^{-1}\left(\left|a_{i_{0}} a_{i_{1}} \cdots a_{i_{k}}\right|\right) \subset \bigcup_{\nu=0}^{k} F_{i_{\nu}}$ for any face $a_{i_{0}} a_{i_{1}} \cdots a_{i_{k}}$ of $Y$. If $f^{-1}\left(\left|a_{i_{0}} a_{i_{1}} \cdots a_{i_{k}}\right|\right)$ is the carrier of a point $p \in S, p$ is contained in at least one $F_{i_{\nu}}$. We thus put $\omega(p)=a_{i_{\nu}}$. Let $N$ be any covering of $S$, and $\tau$ a chain homomorphism discussed in the foregoing sections. On applying Lemma 1 to these $N, \tau$, $\omega$, we see the existence of at least one regular $n$-simplex $\sigma$ in $K(N)$, which implies that at least one member of $N$ intersects all $F_{i}(i=0,1, \cdots, n)$. Suppose that $\bigcap_{i=0}^{n} F_{i}$ were empty. Then the totality of complements $F_{i}^{c}$ of $F_{i}$ gives rise to a covering $N$ of $S$ so that at least one $F_{i}^{c}$ intersects all $F_{j}(j=0,1, \cdots, n)$. This yields a contradiction $F_{i} \cap F_{i}{ }^{c} \neq \phi$. Accordingly $\bigcap_{i=0}^{n} F_{i}$ must be nonempty. Finally it is readily seen that $f(p)=\theta(p)$ if and only if $p \in \bigcap_{i=0}^{n} F_{i}$. 


\section{Main theorem.}

It is the purpose of this section to state and prove a general theorem which contains as special cases all the results in $[1,10,18,19,20,21,22,23,24$, 25, 26]. Before proceeding to the major part of discussion, it will be preceded by preliminary remarks on set-valued maps.

\subsection{Upper semi-continuity and closedness.}

Let $S$ and $X$ be two Hausdorff spaces and $\varphi: S \rightarrow 2^{X}$ be a set-valued map. Unless otherwise stated, all the set-valued maps are those whose image sets $\varphi(p)$ of individual points $p$ are nonempty.

(1) $\varphi$ is said to be upper semi-continuous (abbr. u.s.c.), whenever for each $p \in S$ and each neighborhood $U(\varphi(p))$ there is a neighborhood $V(p)$ such as $\varphi(q) \subset U(\varphi(p))$ for any $q \in V(p)$.

(2) $\varphi$ is said to be closed, whenever the graph $G=\{(p, x) \mid x \in \varphi(p)\}$ is closed in the cartesian product $S \times X$. These two definitions are not necessarily equivalent. But under some additional assumptions they can be made interrelated. Assume now that $S$ be compact. Then we have

Lemma 3. The following two conditions on $\varphi$ are equivalent.

a) Each image set $\varphi(p)$ is compact, and $\varphi$ is u.s.c.

b) There is a compact subset $T$ of $X$ such that $T$ includes all the image sets $\varphi(p)$, and $\varphi: S \rightarrow 2^{T}$ is closed.

Proof. Let $T=\bigcup_{p \in S} \varphi(p)$, then it can be shown, similarly to the case of single-valued maps, that $T$ is compact. We shall next show that $\varphi: S \rightarrow 2^{T}$ is closed. Suppose that $(p, x) \in \bar{G}$. If $\varphi(p) \nexists x$, there would exist two neighborhoods $U(\varphi(p))$ and $W(x)$ in $X$ such that $U(\varphi(p)) \cap W(x)=\phi$. By upper semi-continuity there is a neighborhood $V(p)$ such that $\varphi(q) \subset U(\varphi(p))$ for all $q \in V(p)$. That $(p, x) \in \bar{G}$ implies the existence of $(q, y)$ with $y \in \varphi(q), q \in V(p)$, $y \in W(x)$. But this yields a contradiction $y \in U(\varphi(p)) \cap W(x)$. Therefore $x \in \varphi(p)$, i. e., $(p, x) \in G$, which proves that $G$ is closed in $S \times T$.

Conversely, if b) is fulfilled, any image set $\varphi(p)$ is compact. To see this, suppose that $x \in \overline{\varphi(p)}$ in $T$. Then clearly $(p, x) \in \bar{G}=G$, which implies $x \in \varphi(p)$. Thus $\varphi(p)$ is closed in $T$. Hence follows the compactness of $\varphi(p)$. Now let $U(\varphi(p))$ be a neighborhood of $\varphi(p)$ in $X$. Let $T(V(p))=\widetilde{\bigcup}_{q \in V(p)} \varphi(q)$ for a neighborhood $V(p)$ of $p$, the closure being taken in $T$. Then from the closedness of $\varphi$ follows that $\varphi(p)=\cap T(V(p))$ over all $V(p)$, that is, $U(\varphi(p)) \supset \cap T(V(p))$. Since each $T(V(p))$ is closed in $T$, in view of the compactness of $T$ we see that $U(\varphi(p)) \supset T(V(p))$ for some individual $V(p)$. This completes the proof.

In the sequel the term semi-continuity will be used also in the following 
sense. A real single-valued function $g(p)$ on $S$ is called upper semi-continuous, if the set $\{p \mid g(p)<\lambda\}$ is open in $S$ for every real $\lambda$. It is called lower semicontinuous, if $\{p \mid g(p)>\lambda\}$ is open in $S$ for every real $\lambda$. This concept of semi-continuity should not be confused to that used in connection with a set-valued map.

\subsection{Main theorem.}

A real-valued function $h(y)$ on a convex set $Y$ is said to be quasi-convex, whenever $\{y \mid h(y) \leqq \lambda\}$ is a convex subset in $Y$ for every real $\lambda . h(y)$ is called quasi-concave, if $-h(y)$ is quasi-convex.

In the following all the statements concerning homological concepts like acyclicity or Vietoris maps will be made with regard to a certain fixed field.

THEOREM 4. (i) Let $S, X$ and $Y_{i}(i=1,2, \cdots, m)$ be Hausdorff spaces. Furthermore, $S$ is compact, and each $Y_{i}$ is a compact convex set in which the convex linear combination of finitely many points depends continuously on its coefficients.

(ii) For each $i=1,2, \cdots, m$ there is a real-valued function $h_{i}\left(x, y_{i}\right)$ on $X \times Y_{i}$ which is continuous on $X$ for every fixed $y_{i} \in Y_{i}$ and quasi-convex on $Y_{i}$ for every fixed $x \in X$.

(iii) There is a Vietoris map $f: S \rightarrow Y=Y_{1} \times Y_{2} \times \cdots \times Y_{m}$.

(iv) $\varphi: S \rightarrow 2^{X}$ is an u.s.c. set-valued map whose image sets $\varphi(p)$ are compact and acyclic.

(v) $g(p)$ is a lower semi-continuous real-valued function on $S$.

(vi) $\sum_{i=1}^{m} h_{i}\left(x, f_{i}(p)\right) \geqq g(p)$ for any $p, x$ such as $x \in \varphi(p)$, where $f_{i}(p)$ stands for the projection of $f(p)$ on $Y_{i}$.

If conditions (i)-(vi) are fulfilled, then at some point $\bar{p} \in S$ the corresponding image $\varphi(\bar{p})$ contains a point $\bar{x}$ such that $\sum_{i=1}^{m} h_{i}\left(\bar{x}, y_{i}\right) \geqq g(\bar{p})$ for all $y_{i} \in Y_{i}(i=1,2$, $\cdots, m)$.

Proof. The proof will be done by relating the problem to coincidence points of certain maps in a similar, but more prudent fashion than in the writer's previous results $[\mathbf{2 4}, \mathbf{2 5}]$. We first deal with the case of single-valued $\varphi$. The general case can easily be reduced to this special case in virtue of Theorem 1 due to Vietoris.

(I) Case of single-valued $\varphi$. In this case $\varphi$ is simply a continuous map from $S$ into $X$. Let $A_{i} \subset Y_{i}$ be any finite subsets $(i=1,2, \cdots, m)$, and put

$$
\begin{aligned}
\xi_{i}(p) & =\min _{y_{i} \in A_{i}} h_{i}\left(\varphi(p), y_{i}\right), \\
\xi(p) & =\sum_{i=1}^{m} \xi_{i}(p) .
\end{aligned}
$$

These functions are obviously continuous. With this setup we shall prove 
that at some $p \in S$ the inequality $\xi(p) \geqq g(p)$ holds. To this end, suppose that the contrary were valid. Then the lower semi-continuous $g(p)-\xi(p)$ takes on a positive minimum $m \varepsilon$ on the compact set $S$. Let $\lambda_{i}(p)=\xi_{i}(p)+\varepsilon(i=1,2, \cdots$, $m)$. Then,

$$
\sum_{i=1}^{m} \lambda_{i}(p) \leqq g(p)
$$

identically on $S$. Also, we have $\xi_{i}(p)<\lambda_{i}(p)$ identically on $S$, which implies that for each $p \in S$ there is a point $y_{i} \in A_{i}$ with $h_{i}\left(\varphi(p), y_{i}\right)<\lambda_{i}(p)(i=1,2, \cdots, m)$. Let $C\left(A_{i}\right)$ stand for the convex hull of $A_{i}$ in $Y_{i}$. We now define the continuous map $\theta: S \rightarrow \prod_{i=1}^{m} C\left(A_{i}\right)$ by the formulae:

$$
\begin{aligned}
\theta_{i}{ }^{y}(p) & =\max \left(\lambda_{i}(p)-h_{i}\left(\varphi(p), y_{i}\right), 0\right) \\
\theta_{i}(p) & =\frac{\sum_{y_{i} \in A_{i}} \theta_{i}{ }^{y}(p) y_{i}}{\sum_{y_{i} \in A_{i}} \theta_{i}{ }^{y}(p)} \\
\theta(p) & =\left(\theta_{1}(p), \theta_{2}(p), \cdots, \theta_{m}(p)\right) .
\end{aligned}
$$

$\prod_{i=1}^{m} C\left(A_{i}\right)$ is obviously finite-dimensional. Therefore, by Theorem 3 for $f, \theta$ on $f^{-1}\left(\prod_{i=1}^{n} C\left(A_{i}\right)\right)$, coincidence occurs at some $\bar{p} \in f^{-1}\left(\prod_{i=1}^{m} C\left(A_{i}\right)\right)$ so that $f(\bar{p})=\theta(\bar{p})$, i. e., $f_{i}(\bar{p})=\theta_{i}(\bar{p})(i=1,2, \cdots, m)$. Let $B_{i}=\left\{y_{i} \mid y_{i} \in A_{i}, \theta_{i}{ }^{y_{i}}(\bar{p})>0\right\}$ and $\delta_{i}=\min \theta_{i}{ }^{y_{i}}(\bar{p})$ for $y_{i} \in B_{i}$. Then $f_{i}(\bar{p}) \in C\left(B_{i}\right)$ and $h_{i}\left(\varphi(\bar{p}), y_{i}\right) \leqq \lambda_{i}(\bar{p})-\delta_{i}$ for all $y_{i} \in B_{i}$. Hence, by quasi-convexity, $h_{i}\left(\varphi(\bar{p}), f_{i}(\bar{p})\right) \leqq \lambda_{i}(\bar{p})-\delta_{i}<\lambda_{i}(\bar{p})$. Summing up for all $i$ and appealing to $(*)$ give $\sum_{i=1}^{m} h_{i}\left(\varphi(\bar{p}), f_{i}(\bar{p})\right)<g(\bar{p})$, which contradicts assumption (vi). Accordingly, there is a point $p \in S$ with $\xi(p) \geqq g(p)$, which implies the closed set $F\left(A_{1}, A_{2}, \cdots, A_{m}\right)=\left\{p \mid p \in S, \sum_{i=1}^{m} h_{i}\left(\varphi(p), y_{i}\right) \geqq g(p)\right.$ for $y_{i} \in A_{i}(i=$ $1,2, \cdots, m)\}$ is nonempty for every $m$-tuple of finite sets $A_{i} \subset Y_{i}$; in other words, the family of all these sets $F\left(A_{1}, A_{2}, \cdots, A_{m}\right)$ admits finite intersection property. Thus, in virtue of the compactness of $S$ we have $\cap F\left(A_{1}, A_{2}, \cdots, A_{m}\right)$ $\neq \phi$. This proves the assertion.

(II) General case. By Lemma 3 there is a compact set $T \subset X$ such that $\bigcup_{p \in S} \varphi(p) \subset T$, and $\varphi: S \rightarrow 2^{T}$ is closed. As the graph $G$ is closed in $S \times T$ which is compact, $G$ is also compact. Now let $\widetilde{S}=G$, and replace $S$ by $\widetilde{S}$. A point $\tilde{p} \in \widetilde{S}$ is of the form $\tilde{p}=(p, x)$ with $x \in \varphi(p)$. In view of this fact we shall define: $\tilde{\varphi}(\tilde{p})=x, \tilde{f}(\tilde{p})=f(p), \tilde{g}(\tilde{p})=g(p)$. Except for condition (iii) all the conditions (i)-(vi) are easily seen to be met by this new system. It therefore remains to check on condition (iii). First, $\tilde{f}$ obviously maps $\widetilde{S}$ onto $Y$. For any $y$ the two inverse images $\tilde{f}^{-1}(y)$ and $f^{-1}(y)$ are interrelated in the following way. The projection $\pi(p, x) \rightarrow p$ continuously maps $\tilde{f}^{-1}(y)$ onto $f^{-1}(y)$, 
and $\pi^{-1}(p)=\{p\} \times \varphi(p)$ for any $p \in f^{-1}(y)$. Since $\varphi(p)$ is acyclic for any $p, \pi: \tilde{f}^{-1}(y)$ $\rightarrow f^{-1}(y)$ is a Vietoris map. By Theorem 1, the homology groups for these two spaces are isomorphic. As $f^{-1}(y)$ is acyclic by assumption, so is $\tilde{f}^{-1}(y)$, as was to be shown. Therefore $\tilde{f}: \widetilde{S} \rightarrow Y$ is a Vietoris map, and condition (iii) is also fulfilled. Thus, case (II) reduces to case (I) for $\tilde{\varphi}$.

\subsection{Relation to the previous results.}

It will be noted here that Theorem 4 involves and generalizes the results on economic equilibrium by Arrow-Debreu [1] , Gale [10], McKenzie [18] and the writer [26] as well as the results on game problems by von Neumann $[\mathbf{2 1}, \mathbf{2 2}, \mathbf{2 3}]$, Nash $[\mathbf{1 9}, \mathbf{2 0}]$, the writer $[\mathbf{2 4}, \mathbf{2 5}]$ and others.

In the following as in Theorem 4 wheneyer we say that a certain set is compact and convex, it is always assumed that the convex linear combination of any finite number of its points depends continuously on its coefficients with respect to the given Hausdorff topology.

\section{a) Existence of economic equilibrium.}

Walras (1834-1910) was the first to generally formulate economic equilibrium in terms of supply and demand functions and to conjecture its existence for his model [32], This conjecture had remained unproved, however, for a long time, until recently several authors including this writer, as named before, solved the problem. Most of their results in this line can be reduced to the following type of proposition as given by Gale [10] and this writer [26].

Let $E^{k}$ be a real $k$-space and $S^{k}$ the set of all points $p \in E^{k}$ whose coordinates are nonnegative and add up to unity; the $j$-th coordinate $x_{j}$ of a point $x \in E^{k}$ represents a certain amount of the $j$-th good, while the $j$-th coordinate of $p$ designates the price of the $j$-th good. The inner product in $E^{k}$ will be denoted by $($,$) . (p, x)$ therefore represents the value of a bundle $x$ of goods at a price system $p$. To each given $p \in S^{k}$ correspond some set of scheduled bundles $x$ of goods supplied and some set of those demanded, therefore the excess $\varphi(p)$ of supply over demand. A price system $\bar{p} \in S^{k}$ is said to be an equilibrium price system, if $\varphi(\bar{p})$ contains a bundle $\bar{x}$ of goods whose coordinates are all nonnegative.

Corollary 1. Let the set-valued map $\varphi: S^{K} \rightarrow 2^{E^{k}}$ fulfill the following conditions:

(1) $\varphi$ is u.s.c. and each image set $\varphi(p)$ is compact, convex (more generally, acyclic).

(2) The Walras law holds true, i.e., $(p, x) \geqq 0$ identically for any $x \in \varphi(p)$, $p \in S^{k}$.

Then there is at least one equilibrium price system. 
This result is a much special case of Theorem 4 where $m=1, S=Y=Y_{1}$ $=S^{k}, X=E^{k}, f(p)=p, g(p)=0, h_{1}(x, y)=(x, y)$ and $\varphi: S^{k} \rightarrow 2^{E^{k}}$. The Walras law insures condition (vi). Therefore, by Theorem 4 , at some $\bar{p}$ the image set $\varphi(\bar{p})$ contains a point $\bar{x}$ such that $(p, \bar{x}) \geqq 0$ for all $p \in S^{k}$. This implies, however, that all the coordinates of $\bar{x}$ are nonnegative.

b) Equilibrium points for noncooperative games ([19, 20, 25]).

Let $X_{i}$ be a compact convex set, called the $i$-th player's strategy space, for $i=1,2, \cdots, m$.

There is given a real-valued function $K_{i}\left(x_{1}, \cdots, x_{i}, \cdots, x_{m}\right)$ on the cartesian product $\prod_{i=1}^{m} X_{i}$, called the $i$-th player's payoff, for $i=1,2, \cdots, m$.

Also the following assumptions will be made on each $K_{i}$.

$(\alpha)$ For each fixed $x_{i} \in X_{i}, K_{i}$ is continuous with respect to the $(m-1)$ tuple $\left(x_{1}, \cdots, x_{i-1}, x_{i+1}, \cdots, x_{m}\right)$ of the remaining variables.

( $\beta) \quad K_{i}$ is quasi-concave on $X_{i}$ for each fixed $(m-1)$-tuple $\left(x_{1}, \cdots, x_{i-1}, x_{i+1}\right.$, $\left.\cdots, x_{m}\right)$.

(r) The sum of payoffs $\sum_{i=1}^{m} K_{i}\left(x_{1}, \cdots, x_{i}, \cdots, x_{m}\right)$ is upper semi-continuous on $\prod_{i=1}^{m} X_{i}$

We define $:\left(\bar{x}_{1}, \cdots \bar{x}_{i}, \cdots, \bar{x}_{m}\right) \in \prod_{i=1}^{m} X_{i}$ is called an equilibrium point, if $K_{i}\left(\bar{x}_{1}\right.$, $\cdots, \bar{x}_{i-1}, y_{i}, \bar{x}_{i+1}, \cdots, \bar{x}_{m}$ ) assumes its maximum on $X_{i}$ at $y_{i}=\bar{x}_{i}$ for each $i$.

Corollary 2. Under the assumptions made above there is at least one equilibrium point.

This result is more general than that in [25], because some of assumptions are relaxed, that is, the concavity of payoffs is replaced by their quasi-concavity and the continuity of $\sum_{i=1}^{m} K_{i}$ by its upper semi-continuity.

To show that Theorem 4 implies Corollary 2 attention will be paid to the following simple fact as given before (Lemma 3.1 in [25]): In order for $\left(\bar{x}_{1}, \cdots, \bar{x}_{i}, \cdots, \bar{x}_{m}\right)$ to be an equilibrium point, it is necessary and sufficient that

$$
\sum_{i=1}^{m} K_{i}\left(\bar{x}_{1}, \cdots, \bar{x}_{i-1}, y_{i}, \bar{x}_{i+1}, \cdots, \bar{x}_{m}\right) \leqq \sum_{i=1}^{m} K_{i}\left(\bar{x}_{1}, \cdots, \bar{x}_{i-1}, \bar{x}_{i}, \bar{x}_{i+1}, \cdots, \bar{x}_{m}\right)
$$

for any $y_{i} \in X_{i}(i=1,2, \cdots, m)$.

Now let $S=X=\prod_{i=1}^{m} X_{i}, Y_{i}=X_{i}(i=1,2, \cdots, m)$. Also $\varphi(x)=f(x)=x: X \rightarrow X$ (identity map), $g(x)=-\sum_{i=1}^{m} K_{i}\left(x_{1}, \cdots, x_{i}, \cdots, x_{m}\right), h_{i}\left(x, y_{i}\right)=-K_{i}\left(x_{1}, \cdots, x_{i-1}, y_{i}, x_{i+1}, \cdots, x_{m}\right)$ $(i=1,2, \cdots, m)$. Then all the assumptions of Theorem 4 are fulfilled, in particular, condition (vi) becomes an equality relation, and its assertion implies the existence of a point $\bar{x}=\left(\bar{x}_{1}, \cdots, \bar{x}_{i}, \cdots, \bar{x}_{m}\right)$ satisfying (**). 
c) Minimax theorem ([21, 22, 23, 24] $)$.

Let $K(x, y)$ be a real-valued function on the cartesian product $X \times Y$ of two compact convex sets $X$ and $Y$. If $K(x, y)$ is continuous, quasi-concave on $X$ for fixed $y \in Y$ and continuous, quasi-convex on $Y$ for fixed $x \in X$, then there is a saddle point $(\bar{x}, \bar{y})$ such that $K(x, \bar{y}) \leqq K(\bar{x}, \bar{y}) \leqq K(\bar{x}, y)$ for any $x \in X, y \in Y$.

This result is a special case of b), in which $m=2, X_{1}=X, X_{2}=Y, K_{1}=K$, $K_{2}=-K$.

In a recent paper [30] Sion succeeded in dropping the continuity of the payoff and replacing it by its semi-continuity for this minimax theorem; his method of proof is to more directly appeal to the classical Knaster-KuratowskiMazurkiewicz's lemma. He also emphasizes that the minimax theorem under this relaxed assumption can not be handled by the fixed point method of the writer as developed in $[\mathbf{2 4 , 2 5 ]}$. It will be shown below, however, that a minor modification of the map technique in [24] suffices to meet the Sion's result to the effect that if in c), $K$ is upper semi-continuous in $x$ and lower semi-continuous in $y$ for each fixed value of the other variable, there is also a saddle point.

In virtue of semi-continuity, the function $\inf _{y \in Y} K(x, y)$ is upper semi-continuous on $X$ so that it takes on a maximum $\lambda(-\infty<\lambda<+\infty)$. Likewise $\sup _{x \in X} K(x, y)$ takes on a minimum $\mu(-\infty<\mu<+\infty)$. The assertion will be proved if it is shown that $\lambda \geqq \mu$. Suppose the contrary $\lambda<\mu$ were true, and take an $\eta$ with $\lambda<\eta<\mu . \quad \max _{x \in X} \inf _{y \in Y} K(x, y)=\lambda<\eta$ implies that for each $x \in X$ there is a $y \in Y$ with $K(x, y)<\eta$. Hence, by the upper semi-continuity of $K$ in $x$ and the compactness of $X$, we can choose a finite set $B=\left\{b_{j} \mid j=1,2, \cdots, t\right\}$ $\subset Y$ such that for each $x \in X$ there is a $y \in B$ with $K(x, y)<\eta$. Similarly we can choose a finite set $A=\left\{a_{i} \mid i=1,2, \cdots, s\right\} \subset X$ such that for each $y \in Y$ there is an $x \in A$ with $K(x, y)>\eta$.

Let $L_{i}=\left\{y \mid y \in C(B), K\left(a_{i}, y\right) \leqq \eta\right\}(i=1,2, \cdots, s), M_{j}=\left\{x \mid x \in C(A), K\left(x, b_{j}\right) \geqq \eta\right\}$ $(j=1,2, \cdots, t)$. By semi-continuity, these sets are closed in $C(A)$ and $C(B)$, respectively. As $C(A)$ and $C(B)$ are homeomorphic to some compact convex sets in Euclidean spaces, their topologies may be assumed to be given by some distance functions. Define the nonnegative continuous functions $\sigma_{i}(y)$ on $C(B)$ and $\tau_{j}(x)$ on $C(A)$ as follows:

$$
\begin{aligned}
& \sigma_{i}(y)=\left\{\begin{array}{ccc}
1 & \text { in case } & L_{i}=\phi \\
\operatorname{dis}\left(y, L_{i}\right) & \text { in case } & L_{i} \neq \phi
\end{array} \quad(i=1,2, \cdots, s),\right. \\
& \tau_{j}(x)=\left\{\begin{array}{ccc}
1 & \text { in case } & M_{j}=\phi \\
\operatorname{dis}\left(x, M_{j}\right) & \text { in case } & M_{j} \neq \phi
\end{array} \quad(j=1,2, \cdots, t) .\right.
\end{aligned}
$$


Since $\bigcap_{i=1}^{s} L_{i}=\bigcap_{j=1}^{t} M_{j}=\phi$ by construction, we have $\sum \sigma_{i}(y)>0$ on $C(B)$ and $\sum \tau_{j}(x)>0$ on $C(A)$. This enables us to define the continuous map $\theta: C(A) \times$ $C(B) \rightarrow C(A) \times C(B)$,

$$
\theta(x, y)=\left[\frac{\sum \sigma_{i}(y) a_{i}}{\sum \sigma_{i}(y)}, \frac{\sum \tau_{j}(x) b_{j}}{\sum \tau_{j}(x)}\right] .
$$

By Brouwer's theorem (more generally, Theorem 3) there is a fixed point $(\bar{x}, \bar{y})$ with $(\bar{x}, \bar{y})=\theta(\bar{x}, \bar{y})$. But $\sigma_{i}(\bar{y})>0$ if and only if $\bar{y} \oplus L_{i}$, i. e., $K\left(a_{i}, \bar{y}\right)>\eta$. Likewise $\tau_{j}(\bar{x})>0$ if and only if $K\left(\bar{x}, b_{j}\right)<\eta$. Hence by appealing to quasiconcavity and quasi-convexity, as in Theorem 4 as well as in $[\mathbf{2 4}, \mathbf{2 5}]$ we arrive at a contradiction $K(\bar{x}, \bar{y})<\eta<K(\bar{x}, \bar{y})$, q. e. d.

Though any weakening of assumption as given by Sion is of interest and desirable, the writer does not as yet know whether a similar relaxation is possible in general cases as formulated in Theorem 4.

\section{Existence of equilibrium in linear spaces.}

In this section Corollary 1 to Theorem 4 will be reviewed and put in a more general form to reveal a certain duality relation behind it. This line of extension was first given in [28] for a special case in normed spaces and then in a more general context in [5] for Euclidean spaces and in [29] for normed spaces. The present formulation will be made in locally convex topological linear spaces.

5.1. Let $E$ be a locally convex topological linear space over the field of real numbers; it therefore has a complete system of neighborhoods $U$ of 0 which are convex. Moreover, for an obvious reason, these $U$ may be assumed to be symmetric in the sense that $-U=U$. This originally given linear topology will be referred to as the strong topology. The totality of all continuous linear functionals forms the dual space $E^{*}$ of $E$; its topology is given by adopting all the sets $\left\{p\left|p \in E^{*},\right| p(x) \mid<\varepsilon\right.$ for $\left.x \in \Gamma\right\}$ as neighborhoods of 0 , where $\Gamma$ is a symmetric bounded subset in $E$. Let $(x, p)=p(x)$ for $x \in E, p \in E^{*}$. The weakest linear topology of $E$ in which $(x, p)$ is continuous on $E$ for each fixed $p$ will be called the weak topology of $E$. The corresponding topology in $E^{*}$ will be called the $w^{*}$-topology.

As is well-known, any semi-order $x \geqq y$ in $E$ which is compatible with the linear topological structure completely corresponds to some (strongly) closed convex cone $P$ in $E$ (see, e. g. Bourbaki [4]). With each closed convex cone $P$ in $E$ is paired its dual convex cone $P^{*}=\left\{p \mid p \in E^{*},(x, p) \geqq 0\right.$ for $\left.x \in P\right\}$.

For a locally convex $E$ the following lemmas are well-known. 
Lemma 4. For each closed convex cone $P$ not coinciding with $E$ the corresponding $P^{*}$ contains points other than 0 .

Lemma 5. Let $P$ be a convex cone in $E$. In order that a point $x \in E$ belong to $\bar{P}$ (the closure of $P$ in the strong topology), it is necessary and sufficient that $(x, p) \geqq 0$ for any $p \in P^{*}$.

LEMMA 6. Let $u$ be an interior point of a convex cone $P$ in $E$. Then $(u, p)$ $>0$ for any non-zero $p \in P^{*}$.

As to their proof the standard literature of Bourbaki [4] is recommended.

Definition. A convex cone either in $E$ or in $E^{*}$ is said to be pointed, if it contains no $x$ together with $-x$ except for 0 . For a convex cone $P$ in $E$ let $\left\langle P^{*}\right\rangle$ stand for the set of all non-zero $p \in P^{*}$.

Lemma 7 . If the interior of a convex cone $P$ in $E$ is not empty, then $P^{*}$ is pointed

Proof. Let $p \in P^{*}$, and $p \neq 0$. Take an interior point $u \in P$. Then, by Lemma $6,(u,-p)<0$ so that $-p \notin P^{*}$, proving the pointedness of $P^{*}$.

Lemma 8. If the interior of a convex cone $P$ in $E$ is not empty and does not coincide with $E$, then $\left\langle P^{*}\right\rangle$ is not empty and convex.

Proof. In view of the fact that $P$ and $\bar{P}$ have the same interior, Lemmas 4, 5 imply that $\left\langle P^{*}\right\rangle$ is nonempty. Now let $p, q \in\left\langle P^{*}\right\rangle$. As $p, q \in P^{*}$, $(1-\lambda) p+\lambda q \in P^{*}$ for $0 \leqq \lambda \leqq 1$. Take an interior point $u$. Then, by Lemma 6 , $(u, p)>0,(u, q)>0$ so that also $(u,(1-\lambda) p+\lambda q)>0$. This implies $(1-\lambda) p+\lambda q \neq 0$, and therefore $(1-\lambda) p+\lambda q \in\left\langle P^{*}\right\rangle$.

Let now $P$ be a strongly closed convex cone in $E$ such that $-P \cap P \neq P$; this simply means that $P$ is not a linear subspace of $E$. If $P$ has the nonempty interior, this condition is equivalent to saying that $P \neq E$. We shall show below that any general such $P$ can be approximated by convex cones having the nonempty interiors and not coinciding with $E$. In fact choose an arbitrary $u$ such that $-P \cap P \oplus u \in P$. We take a fixed convex neighborhood $U$ of 0 such that $(-u+U) \cap P=\phi$. For any convex, symmetric neighborhood of $0, V \subset U$, let $Q(V)$ be the convex cone spanned by $u+V$. We put $P(V)=\overline{P+Q(V)} . \quad P(V)$ is a closed convex cone, and contains interior points, because $P(V) \supset Q(V) \supset u+V$. Now we shall prove

LEMiMA 9.

$$
\begin{array}{ll}
\text { (i) } \quad P(V) \mp-u & \text { for any } V \subset U . \\
\text { (ii) } P=\cap P(V) & \text { for all } V \subset U .
\end{array}
$$

Proof of (i). Suppose $-u \in P(V)$. Then we have $-u=x+\lambda(u+e)+a$, for some $x \in P, \lambda \geqq 0, e, a \in V$. Hence, $-u=\frac{x}{1+\lambda}+\frac{\lambda}{1+\lambda} e+\frac{a}{1+\lambda}$, with $\frac{x}{1+\lambda} \in P$. 
Moreover, $\frac{\lambda}{1+\lambda} e+\frac{a}{1+\lambda} \in V$ by convexity. This implies $(-u+V) \cap P \neq \phi$, yielding a contradiction $(-u+U) \cap P \neq \phi$.

Proof of (ii). Suppose $y \in P(V)$ for all symmetric convex $V \subset U$, but $y \notin P$. The first supposition implies that $y=x_{V}+\lambda_{V}\left(u+e_{V}\right)+a_{V}$ for some $x_{V} \in$ $P, e_{V}, a_{V} \in V, \lambda_{V} \geqq 0$. From this relation follows $y-\left(x_{V}+\lambda_{V} u\right)=\lambda_{V} e_{V}+a_{V} \equiv b_{V}$ with $x_{V}+\lambda_{v} u \in P$. In view of $y \notin P$ we choose a symmetric, convex neighborhood $W$ of 0 such that $W \subset U$ and $(y+W) \cap P=\phi$. Thus clearly $b_{V}=$ $\lambda_{V} e_{V}+a_{V} \notin W$.

We first prove that $\lim _{V \rightarrow 0} \lambda_{V}=+\infty$. To this end, it suffices to show that $\lambda_{V}>n-1$ for any $V \subset \frac{1}{n} W$. In fact, since $e_{V}$ and $a_{V}$ belong to the convex set $V$,

$$
\frac{b_{V}}{1+\lambda_{V}}=\frac{\lambda_{V}}{1+\lambda_{V}} e_{V}+\frac{1}{1+\lambda_{V}} a_{V} \in V \subset \frac{1}{n} W
$$

that is, $b_{V} \in \frac{1+\lambda_{V}}{n} W$. However, as $b_{V} \in W$, we must have that $\frac{1+\lambda_{V}}{n}>1$, i. e., $\lambda_{V}>n-1$.

Finally, $b_{V} \in\left(1+\lambda_{V}\right) V$ implies that $b_{V} / \lambda_{V} \in\left(1+1 / \lambda_{V}\right) V$, which entails, in view of the definition of $b_{V}, u+x_{V} / \lambda_{V} \in y / \lambda_{V}-\left(1+1 / \lambda_{V}\right) V$. This result together with $\lim \lambda_{V}=+\infty$ yields a contradiction $-u \in \bar{P}=P$. Therefore, $\cap P(V) \subset P$. In view of $P(V) \supset P$, we have thereby proved (ii).

\subsection{Existence of equilibrium.}

Here we formulate and prove a general version of Corollary 1 in section 4 in a locally convex topological linear space $E$. Let $P$ be a strongly closed convex cone in $E$ such that $-P \cap P \neq P$.

THEOREM 5. Assume that a set-valued $\operatorname{map} \varphi:\left\langle P^{*}\right\rangle \rightarrow 2^{E}$ satisfies the following conditions :

(1) Each image $\varphi(p)$ is nonempty, weakly compact and acyclic.

(2) $\varphi$ is upper semi-continuous as a map: $\left\langle P^{*}\right\rangle \cap L \rightarrow 2^{E}$, for every finitedimensional convex subcone $L$ of $P^{*}$, where the domain and range are viewed in the $w^{*}$-topology of $E^{*}$ and in the weak topology of $E$, respectively.

(3) $\bigcup_{p \in\left\langle P^{*}\right\rangle} \varphi(p)$, that is, the whole image set of $\left\langle P^{*}\right\rangle$ under $\varphi$ is weakly compact.

(4) The Walras law prevails, i.e., $(x, p) \geqq 0$ for $x \in \varphi(p)$ at each $p \in\left\langle P^{*}\right\rangle$. These assumptions together suffice to insure the existence of $a \bar{p} \in\left\langle P^{*}\right\rangle$ such that $\varphi(\bar{p}) \cap P \neq \phi$.

PROOF. This theorem is not directly included in Theorem 4 and will be 
proved below through approximation with the aid of the latter.

Take the $P(V)$ in Lemma 9 obtained by slightly inflating $P$. Since $P(V) \supset$ $P$, we have $P(V)^{*} \subset P^{*}$ for their dual cones. As was noted, all $P(V)$ contain an interior point $u$ in common, but none of them contains $-u$. By Lemma 8 , $\left\langle P(V)^{*}\right\rangle$ is nonempty and convex. Choose any finite subset $F$ of $\left\langle P(V)^{*}\right\rangle$, then the convex hull $C(F)$ is entirely included in $\left\langle P(V)^{*}\right\rangle$. We appeal to Theorem 4 with $m=1, S=Y_{1}=C(F), X=E, h_{1}(x, p)=(x, p)$ for $x \in X_{p} p \in Y_{1}$, $g(p)=0$ for $p \in S$. By this theorem, at some $\bar{p} \in C(F)$ and for some $\bar{x} \in \varphi(\bar{p})$ we have $(\bar{x}, p) \geqq 0$ for any $p \in C(F)$. This result implies that the weakly closed convex cone $P(V, F)=\{x \mid x \in E,(x, p) \geqq 0$ for all $p \in F\}$ intersects $\Delta=$ $\cup_{p \in\left\langle P^{*}\right.} \varphi(p)$ for every finite subset $F$ of $\left\langle P(V)^{*}\right\rangle$. Since $\Delta$ is assumed to be weakly compact, and as $\bigcap_{F} P(V, F)=P(V)$ by Lemma 5, this entails that $P(V) \cap \Delta \neq \phi$ for any $V$. Every $P(V)$ is also weakly closed by Lemma 5 . Hence once more appealing to the compactness of $\Delta$, in the light of Lemma 9, (ii), we arrive at the result $P \cap \Delta \neq \phi$, as was to be shown.

In the above theorem the assumption: $-P \cap P \neq P$ is indispensable. This can be checked by means of several simple examples. Here we give one of them. Let $E=E^{3}, P$ a plane passing through 0 . Then $P^{*}$ is the straight line passing through 0 and perpendicular to $P .\left\langle P^{*}\right\rangle$ is the union of two open half straight lines $l_{1}$ and $l_{2}$. Choose a point $x_{1} \in l_{1}$ and a point $x_{2} \in l_{2}$. We define : $\varphi(p)=x_{1}$ for any $p \in l_{1}$ and $\varphi(p)=x_{2}$ for any $p \in l_{2}$. Though this map fulfills all the conditions of the theorem, $\varphi\left(\left\langle P^{*}\right\rangle\right)$ does not intersect $P$.

\subsection{Applications.}

In this section the implication of Theorem 5 will be noted for several remarkable cases.

a) If $E$ is finite-dimensional, assumption (3) can be dropped so that the remaining assumptions are sufficient for the assertion. To see this, we define another map $\psi:\left\langle P^{*}\right\rangle \rightarrow 2^{E}$ by the formula $\psi(p)=\varphi(p /\|p\|)$ for $p \in\left\langle P^{*}\right\rangle$, where \|\| stands for a norm in $E$. The image of $\left\langle P^{*}\right\rangle$ under $\psi$ is nothing but that of the intersection of $P^{*}$ with the unit sphere so that this image is compact. The remaining assumptions (1), (2) and (4) are also met by $\psi$. Accordingly, by Theorem 5, at some $\bar{p} \in\left\langle P^{*}\right\rangle, \varphi(\bar{p} /\|\bar{p}\|)$ intersects $P$. This completes the proof, since $\bar{p} /\|\bar{p}\| \in\left\langle P^{*}\right\rangle$.

b) Suppose that $E$ is a normed space. \|\| will designate the norm in $E$ as in a). Suppose, furthermore, that $P$ contains interior points. In this case, if $\varphi$ satisfies assumptions (1) and (4) together with a more stringent assumption $\left(2^{\prime}\right)$ (than (2)) $: \varphi:\left\langle P^{*}\right\rangle \rightarrow 2^{E}$ is u. s. c. throughout $\left\langle P^{*}\right\rangle$ in the $w^{*}$-topology, the assertion is also valid in spite of (3) being dropped.

To prove this we shall proceed in a similar way as in a). Take an 
interior point $u \in P$ and let $C_{u}=\left\{p \mid p \in P^{*}, p(u)=1\right\} . \quad C_{u}$ is convex and $w^{*}$ closed in $E^{*}$. If $\varepsilon$-ball $U(u, \varepsilon)=\{x \mid x \in E,\|x-u\| \leqq \varepsilon\}$ is included in $P$, then $p(u) \geqq \varepsilon\|p\|$ for any $p \in P^{*}$. In fact, for any $e \in E$ with $\|e\| \leqq 1$ we have $u-\varepsilon e \in P$ so that $0 \leqq p(u-\varepsilon e)=p(u)-\varepsilon p(e)$. This implies that $\varepsilon\|p\|=\varepsilon$. $\sup _{\|e\| !} p(e) \leqq p(u)$. Therefore, $C_{u}$ is also bounded. Accordingly $C_{u}$ is $w^{*}$ compact. Define now $\psi(p)=\varphi(p / p(u))$ for $p \in\left\langle P^{*}\right\rangle$ in view of the fact that $p(u)>0$ for any $p \in\left\langle P^{*}\right\rangle$ either by Lemma 6 or by $p(u) \geqq \varepsilon\|p\|$ as given above. For a reason similar to that in a) this fulfills assumption (3) as well as the remaining ones. It is also noted that the contracted map $\psi: C_{u} \rightarrow 2^{E}$ can be directly handled by Theorem 4 .

c) Fenchel's theorem ([9]). It is finally noted that a special case of a) implies Fenchel's result on the convex hull of a compact connected set in a Euclidean space $E^{k}$; the theorem reads as follows.

Let $A$ be a compact connected set in $E^{k}$. If a point $x$ in $E^{k}$ belongs to the convex hull $C(A)$ of $A, x$ can be written as a convex linear combination of at most $k$ points of $A$.

Proof. It is well-known that any point belonging to the convex hull of an arbitrary set in $E^{k}$ is representable as a convex linear combination of at most $k+1$ points of this set. Now suppose that $x$ be a positive convex-linear combination of exactly $k+1$ points $a_{0}, a_{1}, \cdots, a_{k}$ of $A$ such that they together span a non-degenerate simplex. Needless to say, only this case deserves consideration. Without loss of generality we may assume $x$ to be 0 . For any non-zero $p \in E^{k}=\left(E^{k}\right)^{*}$, we denote by $\pi(p)$ the linear subspace $\left\{x \mid x \in E^{k}\right.$, $(x, p)=0\}$. At each nonzero $p$, on the both sides of $\pi(p)$ lie some of $a_{i}$, because 0 is an interior point of the simplex. The connectedness of $A$ then implies that $\pi(p) \cap A \neq \phi$ for every nonzero $p$. Denote by $\varphi(p)=C(\pi(p) \cap A)$ the convex hull of $\pi(p) \cap A$ for $p \neq 0$. Let $P$ be the convex cone generated by the point $-a_{0}$. $P$ is obviously closed and fulfills $-P \cap P \neq P$. Then the set-valued map $\varphi:\left\langle P^{*}\right\rangle \rightarrow 2^{E^{k}}$ satisfies conditions (1), (2), (3) together with a stronger condition than $(4):(x, p)=0$ for $x \in \varphi(p)$. By Theorem 5 there exists one $\bar{p} \in\left\langle P^{*}\right\rangle$ such that $\bar{x} \in P$ for some $\bar{x} \in \varphi(\bar{p})$; to be more explicit, $\bar{x}=-\lambda a_{0}, \lambda \geqq 0$. If $\lambda=0$, we have $0=\bar{x} \in \varphi(\bar{p})$. If $\lambda>0$, from $0=(\bar{x}, \bar{p})=$ $-\lambda\left(a_{0}, \bar{p}\right)$ follows $\left(a_{0}, \bar{p}\right)=0$ so that $a_{0} \in \pi(\bar{p}) \cap A \subset \varphi(\bar{p})$. Hence, also $0=$ $\frac{1}{1+\lambda} \bar{x}+\frac{\lambda}{1+\lambda} a_{0} \in \varphi(\bar{p})$. As $\pi(\bar{p})$ is a $(k-1)$-space, 0 can be written as a convex linear combination of at most $k$ points of $\pi(\bar{p}) \cap A$.

The Institute of Social and Economic Research, Osaka University. 


\section{References}

[1] K. J. Arrow and G. Debreu, Existence of an equilibrium for a competitive economy, Econometrica, 22 (1954), 265-290.

[2] E. G. Begle, A fixed point theorem, Ann. Math., 51 (1950), 544-550.

[3] E. G. Begle, The Vietoris mapping theorem for bicompact spaces, Ann. Math., 51 (1950), 534-543.

[4] N. Bourbaki, Éléments de mathématiques, libre V, Espaces vectoriels topologiques, 1953.

[ 5 ] G. Debreu, Market equilibrium, Proc. Nat. Acad. Sci., U.S. A., 42 (1956), 876-878.

[6] S. Eilenberg and D. Montgomery, Fixed point-theorems for multivalued transformations, Amer. J. Math., 68 (1946), 214-222.

[7] K. Fan, Minimax theorems, Proc. Nat. Acad. Sci., U. S. A., 39 (1953), 42-47.

[8] K. Fan, On systems of linear inequalities, in 'Linear inequalities and related systems', (1956), Ann. Math. Studies, No. 38, 99-156.

[9] W. Fenchel, Über Krümmung und Windung geschlossener Raumkurven, Math. Ann., 101 (1929), 238-252.

[10] D. Gale, The law of supply and demand, Math. Scad., 3 (1955), 155-169.

[11] R. A. Hirschfeld, On a minimax theorem of K. Fan, Koninkl. Nederl. Akad. Wet., Proc. Series A, 61 (1958), and Indag. Math., 20 (1958), 470-474.

[12] S. Kakutani, A generalization of Brouwer's fixed point theorem, Duke Math. J., 8 (1941), 457-459.

[13] B. Knaster, C. Kuratowski and S. Mazurkiewicz, Ein Beweis des Fixpunktsatzes für $n$-dimensionale Simplexe, Fund. Math., 14 (1926), 132-138.

[14] H. Kneser, Sur un théorème fondamental de la théorie des jeux, C. R. Acad. Sci. Paris, 234 (1952), 2418-2420.

[15] H.W. Kuhn and A.W. Tucker, eds., Contributions to the theory of games, 1 (1950), Ann. Math. Studies, No. 24.

[16] S. Lefschetz, On the fixed point formula, Ann. Math., 38 (1937), 819-822.

[17] S. Lefschetz, Algebraic topology, Amer. Math. Soc. Colloq. Publ., 27, 1942.

[18] L. W. McKenzie, On equilibrium in Graham's model of world trade and other competitive systems, Econometrica, 22 (1954), 147-161.

[19] J. F. Nash, Equilibrium points in $n$-person games, Proc. Nat. Acad. Sci., U. S. A., 36 (1950), 48-49.

[20] J. F. Nash, Non-cooperative games, Ann. Math., 54 (1951), 286-295.

[21] J. von Neumann, Zur Theorie der Gesellschaftsspiele, Math. Ann., 100 (1928), 295-320.

[22] J. von Neumann, Über ein ökonomisches Gleichungssystem und eine Verallgemeinerung des Brouwerschen Fixpunktsatzes, Ergeb. Math. Kolloq., 8 (1937), 73-83.

[23] J. von Neumann, Theory of games and economic behavior, with O. Morgenstern, Princeton Univ. Press, 1944.

[24] H. Nikaidô, On von Neumann's minimax theorem, Pacif. J. Math., 4 (1954), 65-72.

[25] H. Nikaidô, Note on noncooperative convex games, with K. Isoda, Pacif. J. Math., 5 (1955), Supplement I, 807-815.

[26] H. Nikaidô, On the classical multilateral exchange problem, Metroeconomica, 8 (1956), 135-145; A supplementary note to [26], Metroeconomica, 9 (1957), 
209-210.

[27] H. Nikaidô, On a minimax theorem and its applications to functional analysis, J. Math. Soc. Japan, 5 (1953), 86-94.

[28] H. Nikaidô, On the existence of competitive equilibrium for infinitely many commodities, Tech. Report, 34 (1956), Dept. of Econ., Stanford Univ.

[29] H. Nikaidô, Existence of equilibrium based on the Walras law, ISER Discussion Paper No. 2 (1957), Inst. Soc. and Econ. Research, Osaka University.

[30] M. Sion, On general minimax theorems, Pacif. J. Math., 8 (1958), 171-176.

[31] L. Vietoris, Über den höheren Zusammenhang kompakter Räume und eine Klasse von zusammenhangstreuen Abbildungen, Math. Ann., 97 (1927), 454-472.

[32] L. Walras, Éléments d'économie politique pure, Lausanne, Paris, 1900. 